\title{
BMJ Open Representation of social determinants of health in German medical education: protocol of a content analysis study
}

\author{
Franziska Hommes, ${ }^{1}$ Simon Drees, ${ }^{2}$ Karin Geffert, ${ }^{3}$ Peter von Philipsborn, ${ }^{4}$ \\ Jan M Stratil ${ }^{4}$
}

To cite: Hommes F, Drees S, Geffert K, et al. Representation of social determinants of health in German medical education: protocol of a content analysis study. BMJ Open 2018;8:e020696. doi:10.1136/ bmjopen-2017-020696

- Prepublication history and additional material for this paper are available online. To view these files, please visit the journal online (http://dx.doi. org/10.1136/bmjopen-2017020696).

Received 30 November 2017

Revised 9 May 2018

Accepted 21 May 2018

A Check for updates

C Author(s) (or their employer(s)) 2018. Re-use permitted under CC BY-NC. No commercial re-use. See rights and permissions. Published by BMJ.

${ }^{1}$ Medical Faculty, RWTH Aachen University, Aachen, Germany

${ }^{2}$ Charité - Universitätsmedizin

Berlin, corporate member

of Freie Universität Berlin,

Humboldt-Universität zu Berlin and Berlin Institute of Health,

Berlin, Germany

${ }^{3}$ Medical Faculty, Julius-

Maximilians Universität

Würzburg, Würzburg, Germany

${ }^{4}$ Institute for Medical

Informatics, Biometry and

Epidemiology, Pettenkofer

School of Public Health, LMU

Munich, Muenchen, Germany

Correspondence to

Mr Simon Drees;

simon.drees@charite.de

\section{ABSTRACT}

Introduction Action on the social determinants of health has been key for improving health and prolonging life in the past, and remains so today. Against this background, WHO's Commission on Social Determinants of Health has called for increased efforts to create health workforces trained in recognising, understanding and acting on the social determinants of health. However, little is known about the extent to which current medical education systems prepare graduates for this challenge. We, therefore, aim to analyse the extent to which the medical curriculum in Germany incorporates content on the social determinants of health.

Methods and analysis We will conduct a qualitative and quantitative content analysis of four key document groups which influence medical education in Germany: the national medical catalogue of learning objectives; examination content outlines provided by the German Institute for Medical and Pharmaceutical Examination Questions; the online textbook most widely used for final examination preparation and the full set of questions from two national medical licensing examinations. We will analyse these documents based on a coding system, which we derived deductively from the report of WHO's Commission on Social Determinants of Health as well as other key publications of WHO. We will report quantitative indicators, such as the percentage of text related to social determinants of health for each document type. Moreover, we will conduct a semiqualitative analysis of relevant content.

Ethics and dissemination This study is based on the analysis of existing documents which do not contain personal or otherwise sensitive information. Results from the study will be published in a scientific peer-reviewed journal.

\section{INTRODUCTION}

Article 25 of the Universal Declaration of Human Rights affirms that '[e]veryone has the right to a standard of living adequate for the health and well-being of himself and of his family, including food, clothing, housing and medical care and necessary social services $[\ldots]$.' This right to the highest attainable standard of physical and mental health is not confined to the right to healthcare. On

\section{Strengths and limitations of this study}

- The strength of this study is the comprehensive methodological approach developed for the analysis of medical education, which can be transferred to a variety of settings.

- The analysis will be based on codes derived from key, internationally recognised documents of WHO and will focus on a variety of documents representing content taught and assessed as part of medical education in Germany.

- The analysis is limited to the German medical education system.

- The analysis is limited to the national level. Therefore, curricula of individual universities will not be part of the analysis. The approach of content analysis of key documents is not able to capture the knowledge attained by individual students during their medical studies nor programmes provided by the individual universities which might exceed national expectations.

the contrary, it acknowledges that the right to health embraces a wide range of socioeconomic factors that promote conditions in which people can lead a healthy life, and extends to the underlying determinants of health, such as food and nutrition, housing, access to safe water and adequate sanitation, safe and healthy working conditions, and a healthy environment. The health status of people on a population level is influenced by four crucial pillars: individual biological conditions, medical care, factors of individual behaviour as well as social determinants. ${ }^{1}$ WHO has defined social determinants of health $(\mathrm{SDH})$ as the conditions in which people are born, grow, live, and age and the wider set of forces and systems shaping the conditions of daily life. ${ }^{2}$ There is robust evidence that among the four pillars mentioned above, SDH have the strongest and most far reaching impact on health on a population level, ${ }^{3-6}$ and that the observed 
inequality in health outcomes is to the largest part attributable to inequality in $\mathrm{SDH}^{78}$

Against this background, the WHO Commission on Social Determinants of Health (CSDH) was established in 2005 with the aim to spread knowledge, awareness and acceptance about strategies related to $\mathrm{SDH}$, as well as to integrate these into health policies on a long-term basis. In 2008, the Commission published its report, which emphasises the role of SDH by identifying social inequality as key source for health inequity. Moreover, it calls for action to close the existing social gap within a generation. ${ }^{2}$ Social inequity has been widely identified as a main factor for differences in health statusbetween nations, as well as within nations. ${ }^{29-11}$ The report is considered a key document regarding $\mathrm{SDH}$ in general and has been the basis for previous studies investigating the inclusion of SDH-related contents of medical education. $^{12}$

One part of the Commission's report refers to training of medical and health professionals in terms of SDH. ${ }^{2}$ It emphasises the responsibility, necessity and advantage of a broad understanding of SDH for health professionals and recommends that ' $[\mathrm{e}]$ ducational institutions and relevant ministries make the $[\mathrm{SDH}]$ a standard and compulsory part of training of medical and health professionals. ${ }^{2}$ It stresses that to "develop a workforce that is trained in the $[\mathrm{SDH}]$ ' is a main principle of action for reducing inequalities in health. ${ }^{2} 13$ Thus, recognising their paramount significance, $\mathrm{SDH}$ should be a mandatory subject matter for every medical student and not just an optional elective depending on individual engagement. In this way, medical schools could address their social accountability. ${ }^{14}$

Facing this international background, our study will analyse the role of $\mathrm{SDH}$ in medical education in Germany. As SDH are a cornerstone of social medicine, public and global health, they are frequently taught in these contexts. However, of these three subjects only social medicine and public health are compulsory parts of medical education in Germany. ${ }^{15}$ Despite being a compulsory part of national medical education and assessment since the 1970 s, social medicine still does not receive adequate attention at many medical faculties. Although the importance of global health education has been increasingly recognised, ${ }^{16}$ implementation in Germany remains fragmentary, faces crucial barriers and still depends to a great extent on local commitment of individual teachers or students. ${ }^{17-21}$ Study results from the late 1990s on the role of social medicine in German medical education investigating 32 curricula of medical schools document a substantial neglect of this subject. ${ }^{22}$ There is, to our best knowledge, no indication that representation of SDH in medical education has significantly improved since then, nor has an in-depth study of the representation of SDH in medical education in Germany, considering current teaching and examination materials, been conducted.

\section{RESEARCH AIMS AND QUESTIONS}

The aim of this study is to assess how and to what extent $\mathrm{SDH}$ are represented in key documents that outline curriculum and assessment content in German medical education. To answer this research question, we aim to conduct an analysis of four key document groups, which influence medical education in Germany. In order to answer this overarching research question, we developed a number of subquestions, which are shown in the following. A first, quantitative level of analysis will focus on the absolute and relative frequency of the respective codes in each analysed document (see below) as well as across all documents. These research subquestions are:

- How many references are made in each document type to each SDH code (frequency)?

- How many per cent of the text of each document type refers to each SDH code (relative frequency)?

- How large is the overlap between the different SDH codes?

- What is the distribution of the SDH codes in the four document groups (eg, are codings widely dispersed or clustered in specific parts of the different document types)?

On the second, semiqualitative level of analysis we will focus on the themes and topics covered within the respective codes in each document type (eg, which topics are discussed in the passages of the respective documents assigned to the code 'living conditions').

\section{METHODS}

\section{Overview}

In our study, we analyse four key document groups, covering what medical schools are expected to teach, what medical students use to study for the national medical licensing examination and what they are expected to know when graduating. We analyse the documents using a qualitative and quantitative content analysis, ${ }^{23}$ with a mixed deductive and inductive approach of content structuring and theme analysis, using the software MAXQDA 12 (VERBI, Berlin, Germany).

\section{Materials}

The basis for our assessment of the specific role of SDH in medical education in Germany are four key document groups that outline curriculum and assessment content in German medical education. An outline is provided in online supplementary table 1 in the annex:

1. Germany's national medical catalogue of learning objectives, called National Competency-Based Catalogue of Learning Objectives for Medicine (Nationaler Kompetenzbasierter Lernzielkatalog Medizin; NKLM).

2. An online learning software used by the large majority of German medical students in preparation for the national medical licensing examination, provided by MIAMED(AMBOSS 100-Tage Examenslernplan) (=AMBOSS). 
3. The content outline for the national medical licensing examination, called Content Catalogue for the Second Part of the Examination of Doctors, provided by the German Institute for Medical and Pharmaceutical Examination Questions (IMPP Gegenstandskatalog für den zweiten Abschnitt der ärztlichen Prüfung; IMPPGK2).

4. The full set of questions from two national medical licensing examinations, held in 2016, provided by the German Institute for Medical and Pharmaceutical Examination Questions (Zweiter Abschnitt der ärztlichen Prüfung; EXAM).

Medical studies in Germany normally last 6 years, which typically comprise 2 years of preclinical studies, followed by 3 years of clinical studies and a 1-year internship. Both after the preclinical phase and the clinical phase, all medical students take a written national examination, while an oral examination has to be passed after the 1-year internship to gain the full medical licence. ${ }^{15}$ (Some medical faculties follow a model medical educational programme ('Modellstudiengang') in which the preclinical studies and the clinical studies are more intertwined.) Since the preclinical phase is mostly focused on basic sciences, we limit our analysis to the national medical licensing examination, which has to be passed at the end of the clinical studies.

To improve comparability and harmonise medical curricula, the German Association for Medical Education and the German Medical Faculty Association developed the German Competency-Based Catalogue of Learning Objectives for Medicine (NKLM) based on extensive stakeholder involvement. In it, all core contents of medical curricula are defined in 17 chapters, ranging from communication skills to therapeutic methods. Its aim is to state the profile and competencies for every student graduating from medical studies. It was adopted in 2015 and is currently in a pilot phase. According to a decision by the German Medical Faculty Association, every German medical faculty has been advised to implement the NKLM by aligning it with its own curricula and learning objectives. The NKLM can thus be considered a 'soft law' in German medical education. It is publicly available online and the use for research purposes is allowed under the terms of fair use. ${ }^{24}$

The IMPP-GK2 is the content framework for the national medical licensing examination. As it is the final written examination, the end of the theoretical phase of medical studies and the last time a student will face questions on all medical disciplines, it is an important milestone. The IMPP-GK2 lists 'health problems' and specific diseases that are used to draft questions for the national medical licensing examination. Medical schools are responsible to prepare their students for this examination and the quality of the education at a medical faculty is often judged by the results of its students in the examination. We, thus, consider the IMPP-GK2 to influence curriculum design and student learning behaviour in preparation for the examination.
The document is publicly available online, use for research purposes was considered to be allowed under the terms of fair use. ${ }^{25}$

Based on the content of the IMPP-GK2, the German Institute for Medical and Pharmaceutical Examination Questions (IMPP) develops 320 multiple choice questions for the second part of the state examination, which takes place biannually. The two (at the beginning of our study) most recent examinations, from spring and fall 2016, were selected for our analysis. We acquired the permission to use these examinations for research purposes by courtesy of the IMPP (obtained by FH, 17 January 2017). As noted before, this examination is one of the key milestones in German medical education and determines part of the final grade students receive on graduation. We, thus, consider it to have a strong influence on student learning behaviour. In accordance with previous research, we assume that both the IMPP-GK2 and the examination itself are suitable materials to assess the role of SDH in medical education on a national level. ${ }^{22}$ In particular, the national medical licensing examination is the only standardised assessment taken by all medical students in Germany, therefore, it is the only examination that could ensure every medical student is evaluated on basic SDH-related content.

AMBOSS is a learning software, which provides a 100-day learning plan, specifically developed to prepare medical students for the national medical licensing examination. AMBOSS is the most popular online learning system, most recently used by $95 \%$ of medical students in the preparation for the national medical licensing examination (number according to MIAMED (on personal correspondence, 9 January 2018)). User access for medical students is provided through a 'campus licence' by the majority of German medical faculties (31/38). The widespread use of the AMBOSS learning software was the main reason to select it as a key document, as it ensures that almost every student is exposed to its content. Unlike medical textbooks, AMBOSS's content is specifically tailored to the contents of the national medical licensing examination (which might differ in depth and focus from general medical textbooks). Besides the 100-day learning plan, AMBOSS provides an online reference database for medical students and practising doctors. Of note, our analysis is restricted to the content of the 100-day learning plan and does not cover the remaining content included in AMBOSS, which has a broader scope and also includes continuing medical education material intended for practising physicians. We acquired the permission to analyse the AMBOSS content from MIAMED (obtained by FH, 26 May 2017).

This study does not investigate the individual interests of medical students in SDH or educational opportunities offered by individual medical schools, but the representation of SDH attributed on a national level. We chose the before-mentioned four key document groups based on the assumption that they adequately represent said national-level learning objectives. We also assume that 
they have great influence on the design of curricula and student learning behaviour. ${ }^{26-28}$

\section{Development of coding system}

In order to identify and define descriptors of the SDH codes, such as 'reducing social and health inequities through action on SDH' or 'raising awareness on SDH and health equity among health professionals', we conducted a manual search of WHO main website and the web sites of the respective WHO regional organisations on key documents on SDH. ${ }^{2329-36}$ We conducted a focused literature review to inform the overall research design as well as the background and discussion section of our study. For the literature search, we used a PubMed search syntax based on SDH and Medical Education as core search concepts (the full syntax is provided in the annex). The search yielded 95 results, which were screened by one study author ( $\mathrm{FH}$ or $\mathrm{PvP})$ at title and abstract level using the systematic review screening software Rayyan (Qatar Computer Research Institute, Qatar). Fifty-three relevant full texts were identified, which were screened and reviewed by one study author (FH, PvP, KG, SD or JMS). During the course of the study, further relevant publications were identified and included.

To further identify and define descriptors of SDH, two authors (JMS, FH) manually searched WHO website for key documents on SDH. ${ }^{23}{ }^{29-36}$ The documents were manually screened by the same authors on whether they provided substantive information on SDH.

To develop a coding system for our analysis, we first built a skeletal coding system in a deductive process. As a starting point, we used the final report of the CSDH 'Closing the gap in a generation: Health equity through action on the SDH' (hereafter named the CSDH report), with 11 thematic chapters forming a preliminary coding system. This recent report is a highly influential and widely recognised document covering SDH and containing explicit reference to medical education and training. One additional code (labelled 'SHD in general') was created to capture text pertaining to SDH in general, while not being specific to any of the 11 individual SDH. In a next step, two authors (JMS, FH) assessed the CSDH report and other key WHO documents and subsumed emerging concepts and themes as descriptors under the 12 main codes. In part, we renamed the codes to capture the broad literature-based concepts subsumed under them. For example, the code based on chapter 13 'Gender equity' was broadened to 'Non-discrimination' on the basis of other key WHO documents to also include discrimination and inequities based on individual or population characteristics other than gender. In order to reduce overlap between the codes, an iterative process of (1) exploration of concepts and codes was conducted, (2) assessment of potential overlaps, (3) rearrangement and subsumption of concepts. For example, we moved aspects of affordability and financial risk protection from the code universal health coverage to healthcare financing to reduce overlap among these codes. The process was reviewed and discussed within the whole research team to establish consensus.

Definitions of these 12 codes were derived from the $\mathrm{CSDH}$ report or other key WHO documents focused on SDH by either extracting a given definition or summarising and subsuming the central themes and aspects. This process was conducted primarily by three authors (JMS, PvP, FH) and was discussed within the research team.

Besides these 12 thematic codes, the two auxiliary codes were created based on the iterative process: One code, labelled 'Explicit', for passages which mention SDH explicitly; and a second code, labelled 'Socioeconomic status and health' for passages in which (1) the interaction of socioeconomic status in terms of income, occupation and education status is discussed (eg, impact of poverty on disease risks) or (2) inequalities and/or inequities within a given population are discussed (eg, difference in life expectancy between two federal states of Germany). The second auxiliary code was primarily added in order to conduct a content analysis on the role of socioeconomic status and health as core elements of the concepts of SDH across all $12 \mathrm{SDH}$ codes. This second auxiliary code is primarily meant to facilitate the quantitative content analysis of the documents on the basis of key cross-cutting aspects in the discussion on $\mathrm{SDH}$, for example, the role of poverty and inequality and/or inequity.

The core and auxiliary codes are displayed in table 1 . Online supplementary table 2 in the annex provides the definitions and descriptors as developed in the process described above.

\section{Development of a coding guideline}

In order to reduce intercoder subjectivity, we developed a coding guideline with specific coding rules for each code. For this purpose, we first defined a preliminary coding guideline, containing the label and definition of each code, as well as inclusion and exclusion criteria.

We used this preliminary coding guideline to code a sample of all four data sources (the contents of 2 days of the 100-day learning software of AMBOSS, one chapter of the NKLM, the whole of IMPP-GK2 and one-third of one of the two licence examinations). The coding was conducted by one to five analysts and discussed afterwards by at least three analysts. Prototypical text passages for the respective codes were added to the coding guideline as anchor examples. For text passages where the assignment to a code remained unclear, this conflict was solved in discussion by at least three authors, and the coding guidelines were revised accordingly. After approximately $1 / 8$ of the material of all four data sources had been coded, the process of coding worked smoothly with a high level of intercoder agreement and as no new unclear cases emerged, we assumed that data saturation was reached and the coding guideline to be ready for application. This full coding guideline is provided in the annex in online supplementary tables 3-8. 
Table 1 List of SDH codes and the documents they were derived from

\section{SDH codes}

1. $\mathrm{SDH}$ in general

\section{CSDH report}

Part 1: Setting the scene for a global approach to health equity
Other key WHO

documents

232930364243

2329303236

2. Early childhood development and Equity from the start (chapter 5) education

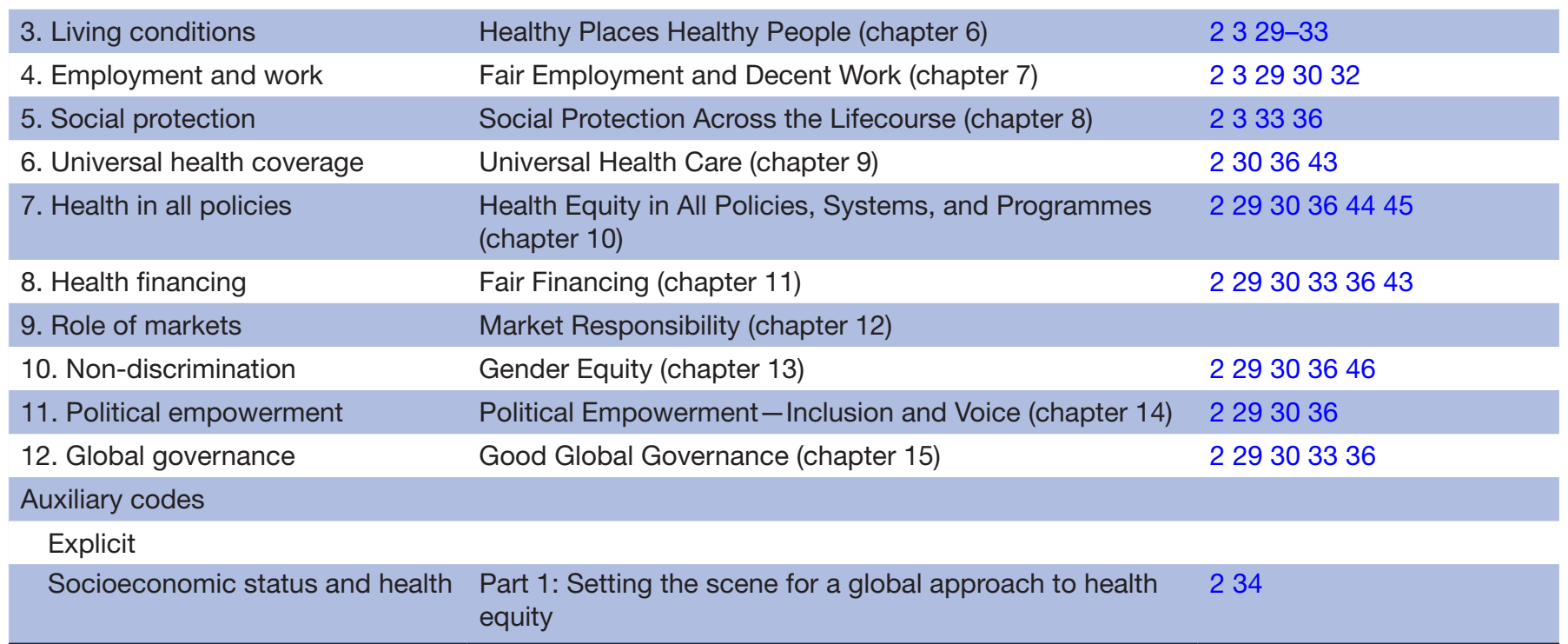

CSDH, Commission on Social Determinants of Health; SDH, social determinants of health.

Moreover, in order to maximise consistency and reproducibility, we developed general coding rules applicable to all codes, covering formal issues. These general coding rules underwent the same testing and revision process as the content-related, code-specific coding guidelines. See the annex for general coding guidelines (online supplementary table 3), document-specific coding guidelines (online supplementary tables 4-7) and additional code-specific coding guidelines (online supplementary table 8).

\section{Coding}

For the final coding of our data, two analysts will independently code the material from the beginning. After finishing the coding of one primary data source, all analysts involved in applying the code will discuss unclear cases and differences in the application of the code. If no solution can be found between the two analysts, conflicts will be discussed between the analysts or the group as a whole. Moreover, we will evaluate the quality of the coding by calculating the degree of intercoder agreement. ${ }^{23}$

\section{Data analysis plan}

We will conduct a quantitative and qualitative analysis. First, we will assess the absolute frequency of the 12 codes across all four data sources combined as well as for the four document groups separately. Next, we will calculate the relative frequency of the different codes within the four document groups by dividing the number of words assigned to the code with the number of words across

Table 2 Document-specific characteristics on which the calculation of the relative frequency of codes within the documents will be based on

\begin{tabular}{|c|c|c|c|}
\hline $\begin{array}{l}\text { National medical catalogue of } \\
\text { learning objectives (NKLM) }\end{array}$ & Online textbook (AMBOSS) & $\begin{array}{l}\text { examination outline (IMPP- } \\
\text { GK2) }\end{array}$ & $\begin{array}{l}\text { National medical licensing } \\
\text { examination (EXAM) }\end{array}$ \\
\hline $\begin{array}{l}\text { Competencies (Kompetenzen) } \\
\text { Subcompetencies } \\
\text { (Teilkompetenzen) } \\
\text { Learning objectives (Lernziele) } \\
\text { Practical examples } \\
\text { (Anwendungsbeispiele, } \\
\text { Beratungsanlass; Krankheit) }\end{array}$ & $\begin{array}{l}\text { Chapters (Lernkarten) } \\
\text { Days of the 100-day learning } \\
\text { schedule }\end{array}$ & $\begin{array}{l}\text { Foreword } \\
\text { Systematical and alphabetical } \\
\text { order of health disorders } \\
\text { Clinical picture, incl. specific } \\
\text { examples }\end{array}$ & $\begin{array}{l}\text { Questions } \\
\text { Case examples (Fallbeispiele) }\end{array}$ \\
\hline
\end{tabular}

IMPP-GK2, Institute for Medical and Pharmaceutical Examination Questions-Gegenstandskatalog für den zweiten Abschnitt der ärztlichen Prüfung; NKLM, Nationaler Kompetenzbasierter Lernzielkatalog Medizin. 
the body of text in the whole documents. Additionally, we will calculate the relative frequency of the codes by building on the specific characteristic of the documents, for example, in the medical examinations, we will calculate the number of questions with an assigned code by the number of questions without an assigned code. ${ }^{37}$ The document-specific characteristics, which will be taken into account when calculating the relative frequency of codes within the documents, are displayed in table 2.

In a next step, two to three authors will conduct a cross-assessment of passages assigned to the respective codes to conduct a theme analysis assessing the content and context of the passages. This will be conducted by first paraphrasing and then generalising the content of each coded section, followed by a reduction and combination of passages with similar content. ${ }^{23}$ Conflicts will be solved through discussion within the research team.

\section{Quality considerations}

In order to reduce subjectivity, all authors (research-specific background of researchers provided in online supplementary table 9 in the annex) jointly reflected, shared, discussed and documented their preconceptions regarding the research subject and expected research findings at the beginning of the research process (Section'Research team and reflexivity' in the annex). While coding, analysing and interpreting the data we will try not to be influenced unduly by these preconceptions and expectations, and carefully consider the possibility of bias arising through them. These are laid out in brief in the annex.

Based on our coding guideline (online supplementary table 3 in the annex) two analysts will conduct the coding independently on all source materials. Conflicts will be solved through discussion between the analysts or the group as a whole. The independent coding followed by discussion is done to fully explore the richness of the data, to control for subjective blurring and to achieve intersubjective certifiability by including and discussing multiple perspectives in the research process. ${ }^{23} 3839$ Moreover, the reproducibility will be measured and reported via the intercoder agreement, as explained above.

\section{Patient and public involvement}

Beyond the authors, no external experts from the public or patient representatives were involved in the development of the study protocol.

\section{ETHICS AND DISSEMINATION}

With our research, we hope to contribute to an improved understanding of the current status of SDH in medical education in Germany, which may help to inform the current ongoing reform process in Germany's medical education system as well as the general debate about public and global health in Germany. ${ }^{40}$ The study has the potential to set a reference point for future studies investigating the role of $\mathrm{SDH}$ in medical education in
Germany. Furthermore, studies on other related thematic contexts in medical education as well as studies on the role of SDH in medical education in other nations could be conducted based on the developed methodology.

This study is based on the analysis of existing data, which does not contain personal or otherwise sensitive information. We, therefore, do not expect any harm for individuals arising from the conduct of our study.

The study was initiated in October 2016 and is currently ongoing. We are planning to start with data analysis once our protocol is published and aim to finish the project by the end of 2018. Results from the study will be published, independent of the nature of the results, in scientific peer-reviewed journals and at conferences. Authorship will be granted only to those who fulfil the authorship criteria recommended by the International Committee of Medical Journal Editors. We will report the results using the Standards for Reporting Qualitative Research checklist. $^{41}$

Acknowledgements We acknowledge support from the German Research Foundation (DFG) and the Open Access Publication Fund of Charité Universitätsmedizin Berlin.

Contributors FH and PvP: conceived the study. PvP, FH and KG: conducted a general literature search on topic. JMS and FH: conducted the literature search of SDH-related WHO documents and preliminary development of coding guidelines. $\mathrm{FH}, \mathrm{PvP}, \mathrm{JMS}, \mathrm{KG}$ and SD: participated in overall study design as well as writing, editing and piloting the protocol. FH: obtained the primary data.

Funding The costs for the analysis software MAXQDA was in part funded by the German Medical Student's Association (bvmd).

Competing interests None declared.

Patient consent Not required.

Ethics approval The research will be undertaken in accordance with the declaration of Helsinki in their respective current versions. As it is a document analysis, no review by an ethics committee was deemed necessary.

Provenance and peer review Not commissioned; externally peer reviewed.

Open access This is an open access article distributed in accordance with the Creative Commons Attribution Non Commercial (CC BY-NC 4.0) license, which permits others to distribute, remix, adapt, build upon this work non-commercially, and license their derivative works on different terms, provided the original work is properly cited, appropriate credit is given, any changes made indicated, and the use is non-commercial. See: http://creativecommons.org/licenses/by-nc/4.0/.

\section{REFERENCES}

1. Tarlov AR. Social determinants of health - the sociobiological transition. London and New York: Routledge Taylor \& Francis Group, 1996.

2. Commission on Social Determinants of Health (CSDH). Closing the gap in a generation: Health equity through action on the social determinants of health. Final Report of the Commission on Social Determinants of Health. Geneva: WHO, 2008.

3. Wilkinson R, Marmot M. Social determinants of health: the solid facts. Copenhagen: WHO Regional Office for Europe, 2003.

4. McKeown T. The modern rise of population. London: Edward Arnold, 1976.

5. Frieden TR. A framework for public health action: the health impact pyramid. Am J Public Health 2010;100:590-5.

6. Pincus T, Esther R, DeWalt DA, et al. Social conditions and selfmanagement are more powerful determinants of health than access to care. Ann Intern Med 1998;129:406-11.

7. World Health Organization (WHO). Equity, social determinants and public health programmes. Geneva: WHO, 2010.

8. Lynch JW, Kaplan GA. Understanding how inequality in the distribution of income affects health. $J$ Health Psychol 1997;2:297-314. 
9. Robert Koch Institut (RKI). Health in Germany. Federal health monitoring system. Berlin: Publication in GermanRKI, 2015.

10. Hofrichter R, Bhatia R. Tackling health inequities through public health practice: theory to action. 2 edn. New York: Oxford University Press, 2010.

11. Marmot M. Social determinants of health inequalities. Lancet 2005;365:1099-104.

12. Hernández-Rincón EH, Pimentel-González JP, Orozco-Beltrán D, et al. Inclusion of the equity focus and social determinants of health in health care education programmes in Colombia: a qualitative approach. Fam Pract 2016;33:268-73.

13. Cole McGrew M, Wayne S, Solan B, et al. Health policy and advocacy for new mexico medical students in the family medicine clerkship. Fam Med 2015;47:799-802.

14. Boelen C, Pearson D, Kaufman A, et al. Producing a socially accountable medical school: AMEE Guide No. 109. Med Teach 2016;38:1078-91.

15. Bundesministerium für Justiz und Verbraucherschutz. Regulations for the licensing of medical doctors in the Version of the 27th June 2002; most recent amendment on the 17th July 2017] Publication in German. Berlin: BMJV, 2002.

16. Rowson M, Smith A, Hughes R, et al. The evolution of global health teaching in undergraduate medical curricula. Global Health 2012;8:35.

17. Bozorgmehr K, Schubert K, Menzel-Severing J, et al. Global Health Education: a cross-sectional study among German medical students to identify needs, deficits and potential benefits (Part 1 of 2: Mobility patterns \& educational needs and demands). BMC Med Educ 2010;10:66.

18. Bozorgmehr K, Menzel-Severing J, Schubert K, et al. Global Health Education: a cross-sectional study among German medical students to identify needs, deficits and potential benefits (Part 2 of 2: Knowledge gaps and potential benefits). BMC Med Educ 2010;10:67.

19. Bruchhausen $\mathrm{W}$, Tinnemann P. [The universities are obliged to act] Publication in German. Deutsches Ärzteblatt 2011;108.

20. Kaffes I, Moser F, Pham M, et al. Global health education in Germany: an analysis of current capacity, needs and barriers. BMC Med Educ 2016;16:304.

21. Knipper M, Baumann A, Hofstetter C, et al. Internationalizing medical education: the special track curriculum 'global health' at justus Liebig University Giessen. GMS Z Med Ausbild 2015;32:Doc52.

22. Schagen U. [Social medicine - suppressed teaching content in medical studies] Revised version of the previous article in: annual of critical medicine 27: health, bureaucracy, managed care. Hamburg 1997, p. 113-136. In: Schagen U, Schleimermacher S, eds. 100 years of social hygiene, social medicine and public health in Germany] Publication in German. Berlin: DGSMP, 2005.

23. Mayring P. Qualitative content analysis: theoretical foundation, basic procedures and software solution. Klagenfurt, 2014.

24. Medizinischer Fakultätentag der Bundesrepublik Deutschland e. V., Gesellschaft für Medizinische Ausbildung (GMA). [National Competency-Based Catalogue of Learning Objectives for Medicine] Nationaler Kompetenzbasierter Lernzielkatalog Medizin (NKLM): Publication in German, 2015.

25. Institut für medizinische und pharmazeutische Prüfungsfragen. Content catalogue for the second part of the examination of doctors (IMPP-GK2)], Publication in German. 4th edn. Mainz: IMPP, 2013.

26. Schauber SK, Hecht M, Nouns ZM, et al. On the role of biomedical knowledge in the acquisition of clinical knowledge. Med Educ 2013;47:1223-35.

27. Van Der Vleuten CP. The assessment of professional competence: Developments, research and practical implications. Adv Health $\mathrm{Sci}$ Educ Theory Pract 1996;1:41-67.
28. McLachlan JC. The relationship between assessment and learning. Med Educ 2006;40:716-7.

29. World Health Organization (WHO). Rio political declaration on social determinants of health - Rio de Janeiro, Brazil. Rio de Janeiro: WHO, 2011.

30. World Health Organization (WHO). World health assembly wha65.8 agenda item 13.6 outcome of the world conference on social determinants of health. Geneva: WHO, 2012

31. World Health Organization (WHO). Social determinants of health sectoral briefing series 1 - housing: shared interests in health and development. Geneva: WHO, 2012.

32. World Health Organization (WHO). Social determinants of health sectoral briefing series 2 - education: shared interests in well-being and development. Geneva: WHO, 2012.

33. World Health Organization (WHO). Social determinants of health sectoral briefing series 4 - social protection: shared interests in vulnerability reduction and development. Geneva: WHO, 2012.

34. World Health Organization (WHO). The economics of social determinants of health and health inequalities: a resource book. Geneva: WHO, 2013.

35. Irwin ASE. Action on the social determinants of health: learning from previous experiences. Discussion Paper 1 (Debates). Geneva: WHO, 2010.

36. World Health Organization (WHO). Global monitoring of action on the social determinants of health: a proposed framework and basket of core indicators - Consultation Paper. Geneva: WHO, 2016.

37. Krippendorff K. Content Analysis - an introduction to its methodology. 2 edn. Thousand Oaks: SAGE publications, 2004.

38. Brod M, Tesler LE, Christensen TL. Qualitative research and content validity: developing best practices based on science and experience. Qual Life Res 2009;18:1263-78.

39. Thomas E, Magilvy JK. Qualitative rigor or research validity in qualitative research. J Spec Pediatr Nurs 2011;16:151-5.

40. Hommes $\mathrm{F}$, von Philipsborn P, Geffert $\mathrm{K}$, et al. [A contribution to the current debate on public and global health in Germany]. Gesundheitswesen 2016;78:126-9.

41. O'Brien BC, Harris IB, Beckman TJ, et al. Standards for reporting qualitative research: a synthesis of recommendations. Acad Med 2014;89:1245-51.

42. Merino BCP, Santaolaya M, Gil A, et al. Integration of social determinants of health and equity into health strategies, programmes and activities: health equity training process in Spain. Geneva: WHO, 2013.

43. Committee on Economic Social and Cultural Rights (CESCR). CESCR General Comment No. 14: The Right to the Highest Attainable Standard of Health (Art. 12) Adopted at the Twenty-second Session of the Committee on Economic, Social and Cultural Rights, on 11 August 2000 (Contained in Document E/C.12/2000/4). Geneva: Office of the High Commissioner for Human Rights, 2000.

44. World Health Organization (WHO). Demonstrating a health in all policies analytic framework for learning from experiences: based on literature reviews from Africa, South-East Asia and the Western Pacific. Geneva: WHO, 2013.

45. World Health Organization (WHO). Practicing a health in all policies approach: lessons for universal health coverage and health equity: a policy briefing for ministries of health based on experiences from Africa, South-East Asia and the Western Pacific. Geneva: WHO, 2013.

46. Kelly MP, Bonnefoy J. Measurement and Evidence Knowledge Network. The social determinants of health: Developing an evidence base for political action. Final Report to World Health Organization Commission on the Social Determinants of Health. Concepción and London: Universidad del Desarrollo \& National Institute for Health and Clinical Excellence, 2007. 